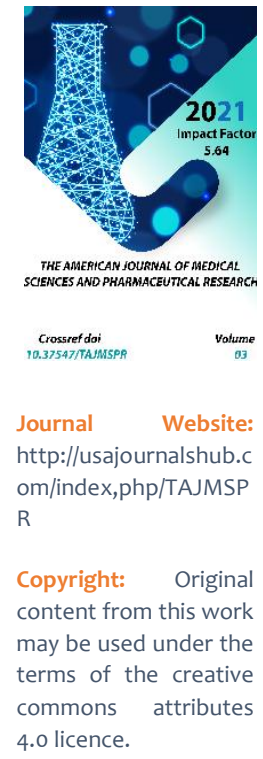

\title{
Clinical Cases Of Hepatitis-Associated Aplastic Anemia
}

Makhmudov Ravshan Barraevich

Bukhara State Medical Institute, Uzbekistan

Safarova Gulnoz Avazkhonovna

Bukhara State Medical Institute, Uzbekistan

\section{ABSTRACT}

The article presents two cases of hepatitis-associated aplastic anemia in the last year. The literature data on the new nosology are presented. The clinical picture, diagnosis and treatment are discussed. The questions of diagnosis and treatment that affect the prognosis are raised. Treatment preference should be given to allogeneic bone marrow transplantation, as it has better results than aggressive immunosuppressive therapy that is difficult for patients to tolerate.

\section{KEYWORDS}

Hepatitis-associated aplastic anemia, immunosuppressive therapy, allogeneic bone marrow transplantation, markers of viral infection.

\section{INTRODUCTION}

Aplastic anaemia (AA) is a rare disorder characterized by pancytopenia and a hypocellular bone marrow. [1] The pathophysiology of the disease is characterized by injury to or loss of pluripotent haematopoietic stem cells, in the absence of infiltrative disease of the bone marrow [2]. The incidence of acquired $A A$ in Europe and North 
America is estimated to be 2 per million populations per year; the incidence is higher in Asia [3].

Hepatitis-associated aplastic anaemia (HAA) is a wellrecognized clinical syndrome in which marrow failure follows the development of hepatitis. 4-9 HAA has been defined as a variant of $A A$, which occurs either concurrently with or within 6 months of an increase in the level of serum alanine aminotransferase (ALT) to at least five times the upper limit of the reference range.4-11 HAA was first described (in two patients) by Lorenz and Quaiser in 1955 [4]. Subsequently, it was documented in $2-5 \%$ and in 4-10\% of cases of $A A$ in the West and the Far East respectively [2, 5]. Thus, HAA accounts for a minor proportion of cases of AA. In a study of 213 children, aged less than 17 years, who had severe $A A$ and who underwent haematopoietic cell transplantation (HCT) or immunosuppressive therapy (IST), the following aetiologies were reported: idiopathic, $80 \%$; posthepatitic, $9 \%$; post-viral infection, $7 \%$; and drugs and other toxins, $4 \% .12$ Hepatitis-associated aplastic anaemia most often develops in adolescent boys and young men, who present with severe pancytopenia 23 months after an episode of acute hepatitis [5]. The marrow failure may be rapid and severe; it is usually fatal if untreated. In this group, there is no documented association with blood transfusions, drugs or toxins and most patients are seronegative for HAV, HBV and HCV [5].

\section{MATERIAL AND METHODS}

Aplastic anemia (AA) is a rare but severe and potentially fatal blood disorder. The reasons for the inhibition of hematopoiesis germs and the development of pancytopenia are not completely clear and are often mixed (exposure to various drugs, chemical compounds, viral agents, autoimmune aggression). In some cases, the etiology remains unknown. Currently, the dominant theory is immune damage to bone marrow cells $[1]$.

In recent years, the term hepatitis-associated aplastic anemia (HAA) has appeared. According to the literature, boys and young men are more likely to get sick, aplasia develops within seven months after hepatitis, and in many in the first month. Overall, 2 to 5 percent of AA cases worldwide are associated with previous hepatitis. More often than not, the involvement of hepatitis $A, B, C$, and $G$ viruses has not been proven. It has been proven that $15 \%$ of patients with seronegative acute liver failure have AA. It is believed that Tlymphocyte activation and cytokine release occurs [2].

In 2002, a study by Japanese doctors was published, which involved 525 children with AA, 61 children were found to have GAAA (12\%). In only one case was hepatitis $A$ virus confirmed, and one each for hepatitis $C$ virus, Epstein-Barr virus (EBV) and cytomegalovirus $(C M V)$. None of them were diagnosed with viral hepatitis $B$ [3].

\section{RESULTS AND DISCUSSIONS}

The GAAA clinic does not differ from the usual AA: anemia, hemorrhagic syndrome, infectious complications. Diagnosis is also similar: complete clinical blood count, including reticulocytes; blood smear; myelogram and trepanobiopsy; cytogenetic blood test; Hem's test or determination of CD55, CD59; examination of liver function tests; viruses: hepatitis A, B, C; Ultrasound of the abdominal organs. $A A$ is often complicated by the development of clonal diseases myelodysplastic syndrome, paroxysmal nocturnal hematuria, or acute myeloid leukemia. It has been noted that such diseases can occur several years after successful immunosuppressive therapy [4]. 
Allogeneic bone marrow transplantation or combined immunosuppressive therapy (IST) are used in the treatment of patients with AA. If there is a donor and there are no contraindications, it is preferable to perform a transplant. According to Japanese researchers, the survival rate after allogeneic transplantation was $85 \%$ at 5.5 years versus $61 \%$ of those who survived after immunosuppressive therapy at 7 years of follow-up. Immunosuppressive therapy is more likely to cause clonal complications. The prognosis for GAAA is poor [5].

In the period November 2018 - April 2019, two patients with GAAA were observed in the hematology department of the Bukhara regional multidisciplinary medical center of the Republic of Uzbekistan.

1. Patient K. A. V., born on January 16, 1985 (33 years old), an industrial climber, was admitted with complaints of general weakness, rapid fatigue. The disease began about 3 months ago: sleep disturbance, loss of appetite, general weakness. A week after the onset of these symptoms, jaundice appeared, the patient was admitted to an infectious diseases hospital with the syndromes of cytolysis (ALT - up to $878 \mathrm{U} / \mathrm{L}, \mathrm{AST}$ - up to $1055 \mathrm{U} / \mathrm{L})$ and cholestasis (GGT - up to $635 \mathrm{U} / \mathrm{L}, \mathrm{ALP}$ - up to $685 \mathrm{E} / \mathrm{L}$, total bilirubin - up to $436 \mu \mathrm{mol}$ / L). He was diagnosed with "Acute hepatitis of unspecified etiology", since the markers of viral hepatitis, autoimmune inflammation were negative, he denied alcohol intake. Took prednisone $50 \mathrm{mg}$ tablets with a gradual dose reduction. A month after discharge, bruises on the body and nosebleeds appeared, in the analyzes a decrease in platelets to $19 \times 109 / \mathrm{L}$ and hemoglobin to $73 \mathrm{~g} / \mathrm{L}$. In the myelogram, there is a sharp decrease in myelokaryocytes and megakaryocytes. Trepanobiopsy results - adipose tissue occupies more than $80 \%$. The purpose of hospitalization in the hematology department of the Republican Clinical Hospital of the Ministry of Health of the Republic of Tatarstan was to conduct immunosuppressive therapy.

Objectively: a state of moderate severity due to shortness of breath with light physical exertion, palpitations, weakness, nosebleeds. The physique is normosthenic. Height $182 \mathrm{~cm}$, weight $76 \mathrm{~kg}$. BMI - $23 \mathrm{~kg} / \mathrm{m2}$. Body surface area according to Dubois is $1.89 \mathrm{mz}$.

Pale skin, two spider veins on the trunk, minor petechial eruptions on the legs. Visible mucous membranes are pale, clean, of moderate moisture. Peripheral lymph nodes are not enlarged. The musculoskeletal system was normal. Lungs - vesicular breathing, wheezing is not heard. NPV - 17 in $1 \mathrm{~min}$. Heart - the boundaries of relative dullness are not expanded. The tones are rhythmic, muffled. BP $-120 / 80 \mathrm{~mm}$ Hg. Art. Pulse -78 beats. in $1 \mathrm{~min}$. The abdomen is symmetrical, participates in the act of breathing, on palpation it is soft, painless. The liver and spleen are not enlarged.

In laboratory tests - severe anemia, leukopenia, thrombocytopenia.

Diagnosed with severe aplastic anemia, first established, associated with hepatitis.

Treatment was carried out: antithymocytic gammaglobulin $1250 \mathrm{mg}$ per 400.0 - physical. solution for 12 hours, concomitant therapy with prednisolone, suprastin, antibacterial therapy (biseptol, fluconazole, ceftazidime, vancomycin, amikacin, ertapenem), replacement therapy with erythrocyte mass, platelet mass, FFP.

Against the background of immunosuppressive therapy with antithymocyte gamma globulin, myelotoxic agranulocytosis, DIC stage II-III syndrome with 
a predominance of the hypocoagulation phase, and spastic colitis were observed. Four months after the onset of the disease, the patient died on the background of severe hemorrhagic syndrome and agranulocytosis, intestinal paresis.

2. Patient B.V. Kh., 25.12.1984 (34 years old), a nurse, was admitted with complaints of general weakness, a periodic increase in body temperature to 39.0, accompanied by chills, regardless of the time of day, the appearance of bruises on the lower extremities of different sizes of different ages, pain in the cervical spine and occipital region.

A year ago I gave birth to my third child, during pregnancy and immediately after giving birth I felt good. She got sick about 8 months ago. Weakness appeared, after 2 weeks - jaundice. A biochemical blood test revealed cytolysis (ALT - $1308 \mathrm{U} / \mathrm{L}, \mathrm{AST}-486 \mathrm{U} / \mathrm{L}$ ), cholestasis (total bilirubin - $348 \mu \mathrm{mol} / \mathrm{L}$, direct bilirubin - 186.1 $\mu \mathrm{mol} / \mathrm{L})$. Markers of viral hepatitis were negative, the patient was admitted to the gastroenterology department, where she was diagnosed with "Autoimmune hepatitis, acute onset with severe cytolysis and hepatocellular failure." Received prednisolone $50 \mathrm{mg}$ in tablets with a gradual dose reduction and omeprazole. Blood tests changed within two months: severe anemia, thrombocytopenia, leukopenia developed. Formed phlegmon in the gluteal region.

Objectively: severe condition due to severe hemorrhagic syndrome (hemorrhagic stroke, ecchymosis on the skin, nose and uterine bleeding), severe weakness, fever up to $39^{\circ} \mathrm{C}$, shortness of breath with light exercise. The physique is normosthenic. Height $162 \mathrm{~cm}$, weight $86 \mathrm{~kg}$. BMI - $33 \mathrm{~kg} / \mathrm{m} 2$. Pale skin, multiple ecchymosis on the skin of the arms, legs, trunk. Visible mucous membranes - pale, multiple petechiae in the oral cavity. Peripheral lymph nodes are not enlarged. The musculoskeletal system was normal. Lungs vesicular breathing, wheezing is not heard. NPV - 17 in $1 \mathrm{~min}$. Heart - the boundaries of relative dullness are not expanded. The tones are rhythmic, muffled. BP - 110/70 mm Hg. Art. Pulse - 98 beats. in $1 \mathrm{~min}$. The abdomen is symmetrical, increased in size due to the subcutaneous fat, participates in the act of breathing, soft, painless on palpation. The liver is enlarged by $2 \mathrm{~cm}$, painless, even. The spleen is not enlarged.

In laboratory tests, severe anemia (hemoglobin decreased to $50 \mathrm{~g} / \mathrm{I}$ ), leukopenia (up to $0.2 \times 109 / \mathrm{I}$ ), thrombocytopenia (up to $5.0 \times 109 / I)$. In the myelogram - the absence of megakaryocytes and a decrease in the cells of the myelocytic lineage, relative lymphocytosis. The patient refused to perform trepanobiopsy. A slight increase in the titer of antibodies to the microsomal fraction of the liver and kidneys was found. Immunophenotyping of the bone marrow - hypocellular bone marrow, the granulocytic region is sharply narrowed. Cytogenetic study of bone marrow - no dividing cells were found.

Was diagnosed "with supersevere aplastic anemia, hepatitis-associated. Hemorrhagic stroke in the form of subarachnoid hemorrhage of the left occipital lobe, left cerebellar hemisphere, left frontal lobe. DIC syndrome stage II-III, chronic course. Polycystic liver and kidney disease. Obesity of the 1st degree. Cholelithiasis. Left shin wound after opening the hematoma".

The patient was hospitalized several times in the $\mathrm{RCH}$, where she was treated with cyclosporine, dicinone, antibacterial and substitution therapy. For maintenance therapy, she was transferred to the Central District Hospital, where she died with symptoms of cardiovascular failure against the background of myelotoxic agranulocytosis. 


\section{CONCLUSIONS}

The two cases of GAAA described above share common features: negative markers of viral hepatitis, a short period between the development of hepatitis and aplastic anemia (1-2 months), the development of pancytopenia during the period of decreasing the dose of prednisolone, and the lack of response to IST. Unfortunately, in both cases, due to the lack of donors, allogeneic bone marrow transplantation was not performed.

\section{REFERENCES}

1. Kosterina A.V., Nazipova Z.M., Marapov D.I. The effectiveness of the use of immunosuppressive therapy and splenectomy in the treatment of patients with aplastic anemia on the example of the experience of the Department of Hematology of the $\mathrm{RCH} / /$ Practical Medicine. - 2016. - No. 4 (96). - S. 68-72. [in russian]

2. Brown K.E., Tisdale J., Barrett A.J., et al. Hepatitis-associated aplastic anemia // N. Engl. J. Med. - 1997. — 336. - P. 1059.

3. Osugi Y., Yagasaki H., Sako M., et al. Antithymocyte globulin and cyclosporine for treatment of 44 children with hepatitis associated aplastic anemia // Haematologica. - 2007. - 92. - P. 1687.

4. Ohara A., Kojima S., Okamura J., et al. Evolution of myelodysplastic syndrome and acute myelogenous leukaemia in children with hepatitis-associated aplastic anaemia // Br. J. Haematol. — 2002. - 116. - P. 151.

5. Савченко В.Г. Клинические рекомендации по лечению апластической анемии // Национальное гематологическое общество. - 2014. C. 8-22. 